\title{
Atuação do profissional enfermeiro frente ao paciente portador de Lesão Por Pressão
}

\author{
Performance of the professional nurse in relation to the patient with Pressure Injury
}

Actuación del enfermero profesional en relación al paciente con Lesión por Presión

José Willian Lima da Silva ORCID: https://orcid.org/0000-0003-2428-5082 Centro Universitário de Juazeiro do Norte, Brasil E-mail: josewillianlima12@gmail.com

Geovanna Renaissa Ferreira Caldas ORCID: https://orcid.org/0000-0001-9820-309X Centro Universitário de Juazeiro do Norte, Brasil E-mail: geovannacaldas@hotmail.com

Francisco de Assis Moura Batista ORCID: https://orcid.org/0000-0003-2403-4830 Centro Universitário de Juazeiro do Norte, Brasil E-mail: assisbaptista13@gmail.com

Lohany Stéfhany Alves dos Santos ORCID: https://orcid.org/0000-0003-3440-740X Hospital Maternidade São Francisco de Assis, Brasil E-mail: lohanystefhany@gmail.com

Maria Paloma Lima Sousa

ORCID: https://orcid.org/0000-0002-3029-0307

Secretaria Municipal de Saúde do Barro, Brasil E-mail: paloma.lima2017@outlook.com

Victor Augusto Moura

ORCID: https://orcid.org/0000-0003-4239-5525 Faculdade de Enfermagem Nossa Senhora das Graças, Brasil E-mail: vito.moura1901@gmail.com

Eva Natalina Ferreira Costa

ORCID: https://orcid.org/0000-0003-2673-6967 Universidade Federal do Estado do Rio de Janeiro, Brasil E-mail: evacostaferreira@gmail.com

Ivo da Silva Soares

ORCID: https://orcid.org/0000-0001-7163-3073 Instituto Brasileiro de Medicina e Reabilitação, Brasil

E-mail: ivossoares23@gmail.com

Flávia Martins Branco

ORCID: https://orcid.org/0000-0003-3484-8555 Universidade Federal do Estado do Rio de Janeiro, Brasil E-mail: flaviabrancoenf@gmail.com

Dayane Martins da Silva

ORCID: https://orcid.org/0000-0002-0193-4417 Universidade Federal do Estado do Rio de Janeiro, Brasil

E-mail: camposcampos_dayane@hotmail.com

Adriana Soares Santos

ORCID: https://orcid.org/0000-0002-4956-1015

Faculdade Estácio São Luis Maranhão, Brasil

E-mail: adryannasoares353@gmail.com

Jackson da Silva

ORCID: https://orcid.org/0000-0002-5373-9699 Centro Universitário UNIFBV, Brasil

E-mail: jacksonsilva10@bol.com.br

Emanuel Cardoso Monte

ORCID: https://orcid.org/0000-0002-4719-7168

Centro Universitário de Juazeiro do Norte, Brasil

E-mail: emanoelcm@gmail.com

Cintia Nadhia Alencar Landim

ORCID: https://orcid.org/0000-0002-8189-9323

Centro Universitário de Juazeiro do Norte, Brasil E-mail: nadhia_landim@hotmail.com

Maria Elisa Regina Benjamin de Moura

ORCID: https://orcid.org/0000-0003-3145-4307

Centro Universitário de Juazeiro do Norte, Brasil E-mail: elisareg@gmail.com 


\title{
Cicero Rafael Lopes da Silva \\ ORCID: https://orcid.org/0000-0001-8819-5380 Centro Universitário de Juazeiro do Norte, Brasil E-mail: rafael.lopes@unijuazeiro.edu.br \\ Dayse Christina Rodrigues Pereira Luz ORCID: https://orcid.org/0000-0002-5719-3574 Faculdade de Medicina do ABC, Brasil E-mail: dayseluz.dcrp@gmail.com
}

\begin{abstract}
Resumo
Objetivou-se analisar as produções científicas sobre a assistência de enfermagem frente ao paciente portador de LPP, com a finalidade de se ter uma compreensão acerca dessa assistência prestada. Trata-se de uma revisão integrativa da literatura, de cunho descritivo e abordagem qualitativa, através da busca nas bases de dados Literatura Latino-Americana e do Caribe em Ciências da Saúde e na Medical Literature Analysis and Retrieval Sistem Online via Biblioteca Virtual em Saúde, com utilização dos Descritores em Ciências da Saúde: Enfermeiro, Assistência e Lesão Por Pressão em associação ao operador booleano AND, obtendo a totalidade de 10 artigos para compor o estudo. Pode-se notar a importância do conhecimento e da assistência do profissional de enfermagem frente ao paciente portador de LPP. A importância da assistência qualificada do enfermeiro baseada não somente no conhecimento empírico, mas principalmente no contexto científico, se mantendo atualizado para um melhor entendimento sobre a forma de prevenir e tratar uma LPP, de maneira com que não ocorra percas acima do normal para a família e para o paciente que necessita desses cuidados.
\end{abstract}

Palavras-chave: Enfermeiro; Assistência; Lesão por pressão.

\begin{abstract}
The objective of this study was to analyze the scientific productions on nursing care for patients with PPL, in order to have an understanding of the care provided. It is an integrative literature review, of a descriptive nature and qualitative approach, through the search in the databases of Latin American and Caribbean Literature in Health Sciences and in the Medical Literature Analysis and Retrieval System Online via Virtual Health Library, using Health Sciences Descriptors: Nurse, Assistance and Pressure Injury in association with the Boolean operator AND, obtaining a total of 10 articles to compose the study. It can be noted the importance of the knowledge and assistance of the nursing professional in the face of the patient with LPP. The importance of qualified nurse assistance based not only on empirical knowledge, but mainly in the scientific context, keeping up-to-date for a better understanding on how to prevent and treat a PPL, so that there are no more than normal losses for the family. and for the patient who needs this care.
\end{abstract}

Keywords: Nurse; Assistance; Pressure injury.

\section{Resumen}

El objetivo de este estudio fue analizar las producciones científicas sobre el cuidado de enfermería al paciente con LPP, con el fin de conocer el cuidado brindado. Se trata de una revisión bibliográfica integradora, de carácter descriptivo y abordaje cualitativo, mediante la búsqueda en las bases de datos de Literatura Latinoamericana y Caribeña en Ciencias de la Salud y en el Sistema de Análisis y Recuperación de Literatura Médica en Línea vía Biblioteca Virtual en Salud, utilizando Descriptores de Ciencias de la Salud: Enfermera, Asistencia y Lesión por Presión en asociación con el operador booleano Y, obteniendo un total de 10 artículos para componer el estudio. Se puede señalar la importancia del conocimiento y asistencia del profesional de enfermería ante el paciente con LPP. La importancia de una asistencia de enfermería calificada basada no solo en el conocimiento empírico, sino principalmente en el contexto científico, manteniéndose actualizado para una mejor comprensión de cómo prevenir y tratar una LPP, de modo que no haya más pérdidas que las normales para el familia. y para el paciente que necesita esta atención.

Palabras clave: Enfermera; Asistencia; Lesión por presión.

\section{Introdução}

Muitas mudanças na sociedade vêm a ocorrer por causa dos avanços tecnológicos e científicos, que acabaram aumentando a expectativa de vida da população, consequentemente trazendo à tona problemas crônicos e traumas que demandam um cuidado com maior complexidade e sua grande maioria em ambientes hospitalares. E é nessas condições de hospitalizações mais prolongadas o paciente torna-se mais suscetível a diversas complicações de saúde, entre essas destacamos a Lesão Por Pressão (LPP) (Sousa \& Faustino, 2019).

LPPs são áreas de destruição tecidual produzida pela compressão da pele contra as proeminências ósseas, com maior incidência de acometimento dos ossos da região sacra, trocantérica, isquiática e calcânea, por conta que a superfície de contato 
tem duração por um tempo mais prolongado, o que acarreta em um prejuízo no fornecimento de sangue e consequentemente de nutrientes aos tecidos, levando a insuficiência vascular, anóxia tecidual e morte das células (Almeida et al., 2019).

A lesão por sua possível derivação, desperta uma atenção maior para possíveis pontos que podem vir a se associar com essa derivação, levando a uma complicação do quadro clínico da lesão, entre alguns desses pontos, nós vamos identificar os dispositivos médicos em seu mau uso, os coxins quando não utilizado corretamente, até mesmo a permanência do decúbito prolongado em uma posição elevando a pressão na região (Adriani et al., 2019).

Destacado em diversos estudos como um fenômeno complexo, tais lesões dependem de fatores intrínsecos e extrínsecos, sendo um dos principais fatores de risco a imobilidade do paciente hospitalizado ou domiciliado. Tal agravo à saúde reflete-se como um relevante problema de saúde pública, capaz de gerar grande desconforto biopsicossocial para o paciente e familiares, além do aumento de custos no tratamento e a necessidade de maiores cuidados de enfermagem, prolongando a sua hospitalização, o que acarreta maiores riscos para a saúde do enfermo (Galvão, 2017).

Apesar de existirem diretrizes internacionais que direcionam a prevenção e o tratamento da LPP como a National Pressure Injury Advisory Panel (NPIAP), é oportuno mencionar que poucos enfermeiros as utilizam, seja por desconhecimento ou insuficiência de materiais e equipamentos necessários para essa prática (Lima et al., 2017).

Em abril de 2013, o Ministério da Saúde instituiu o Programa Nacional de Segurança do Paciente (PNSP), por meio da Portaria MS/GM n n $^{\circ}$ 29/2013, na qual um dos objetivos destacados é o monitoramento da incidência de LPP, objetivando minimizá-la em pacientes hospitalizados (Gonçalves et al., 2015).

Durante o processo de criação de estratégias para prevenção das lesões, o âmbito da Atenção Básica (AB) é considerado de extrema importância, pois o mesmo permite um acompanhamento contínuo na Estratégia de Saúde da Família (ESF), destacando-se dessa forma, o profissional enfermeiro, estabelecendo essa função de minimizar os danos decorrentes da lesão (Silva et al., 2019).

Para a realização do tratamento de LPPs torna-se necessária a atuação de uma equipe multiprofissional, neste sentido, destacam-se os enfermeiros como agentes especiais durante todo o processo de prevenção e tratamento de lesões, estes que devem manter-se atualizados para atuação no contexto de saúde apresentado, efetivando a prevenção, a cicatrização e a readaptação dos pacientes ao processo de saúde doença, definindo fatores que interfiram e venham a retardar todo o tratamento, por meio de condutas para eliminação da pressão continua da pele e demais fatores, como cisalhamento e hiperemia par que se consiga evitar o agravamento da lesão ou até mesmo o surgimento desta (Lima et al., 2017).

Um programa de prevenção orientado pela avaliação de riscos reduz em até $60 \%$ o aparecimento dessas lesões, sendo que entre as ferramentas válidas para o uso elementar de avaliação de risco de LPP, o profissional encontra as escalas de Norton e de Braden (Nunes et al., 2019).

Diante do exposto em relação à LPP e o papel do profissional de enfermagem na prevenção e assistência ao cuidado, destaca-se a necessidade de um amplo conhecimento teórico-prático do passo a passo da assistência de enfermagem ao portador dessa patologia, carecendo então, de um profissional que esteja capacitado para atuar no cuidado dessas lesões.

Neste sentido, objetivou-se analisar as produções científicas sobre a assistência de enfermagem ao paciente portador de LPP.

\section{Metodologia}

Trata-se de uma revisão integrativa da literatura, de cunho descritiva e abordagem qualitativa, desenvolvida nas seguintes etapas: identificação do tema e seleção da questão norteadora da pesquisa; Investigação da produção científica que atenda a questão norteadora, conforme critérios de inclusão e exclusão; Coleta de dados; Análise dos dados e por fim elucidação dos dados e apresentação da revisão. 
A revisão integrativa é a mais ampla abordagem metodológica referente às revisões, permitindo a inclusão de estudos experimentais e não experimentais para uma compreensão completa do fenômeno analisado (SOUZA, 2010).

$\mathrm{Na}$ perspectiva de obter dados apropriados ao propósito, realizou-se a busca nas bases de dados Literatura LatinoAmericana e do Caribe em Ciências da Saúde (LILACS) e na Medical Literature Analysis and Retrieval Sistem Online (MEDLINE) via Biblioteca Virtual em Saúde (BVS), com utilização dos Descritores em Ciências da Saúde (DECS): Enfermeiro, Assistência e Lesão Por Pressão em associação ao operador booleano AND, durante os meses Janeiro e Fevereiro de 2020.

Entre os critérios de elegibilidade, foram incluídos: artigos originais, disponibilidade do artigo na íntegra, com publicação nos idiomas português e espanhol, publicados no período de 2015 a 2019 que abordassem a temática do estudo e possibilitaram responder à questão norteadora desta pesquisa e seleção do título que contenha referências aos descritores usados na pesquisa.

Foram excluídos estudos com animais e/ou inconclusivos, estudos que não foram disponibilizados na íntegra, idiomas diferentes do critério de inclusão, título que não condiziam com os descritores, além daqueles que apresentaram duplicidade na pesquisa e texto sem real relevância para o escopo do estudo.

Esta fase de seleção foi realizada pelos pesquisadores de forma independente, inicialmente por meio da adequação dos títulos e resumos à proposta do estudo onde após leitura, os dados foram inseridos em um instrumento elaborado pelos autores.

Dos estudos foram extraídas informações como: autor, ano, objetivo e amostra onde esses traziam dados referentes a cada artigo usado durante a elaboração do estudo, sendo tais informes inseridos no quadro 01 . O quadro 02 expõe os principais achados de cada artigo, sendo utilizada a associação correspondente do número do artigo no quadro 01 ao número do achado no quadro 02. Na etapa de avaliação, foi realizada uma análise crítica dos estudos, a interpretação dos dados baseando-se na literatura vigente levando a discussão entre os autores.

\section{Resultados}

Realizou-se a busca das referências nas literaturas e a Figura 1, seguinte, ilustra graficamente como foi decorrida essa busca e seleção:

Figura 1: Representação gráfica da aplicação de critérios de inclusão e exclusão.

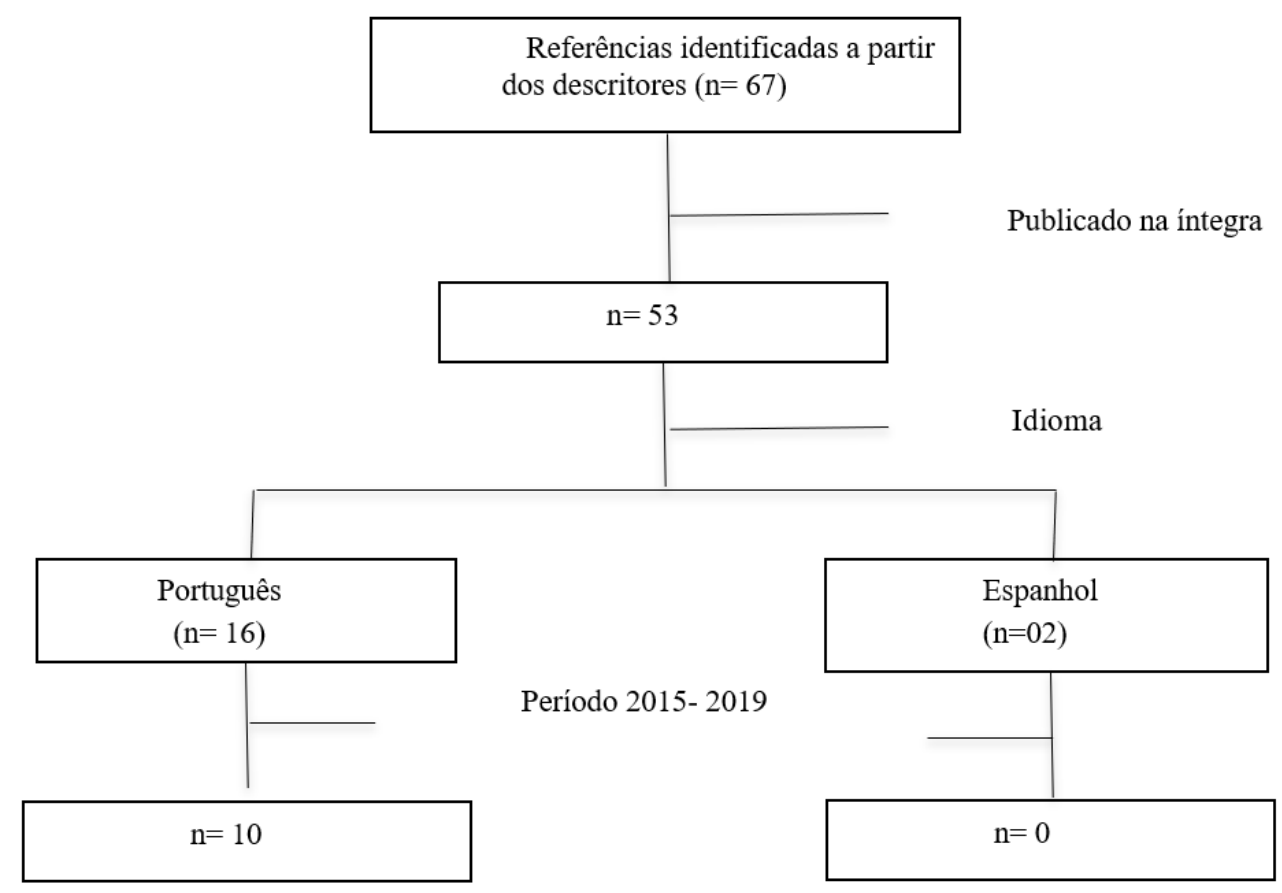

Fonte: Elaboração própria dos autores. 
A Figura 1 demonstra que apesar de terem sido encontradas 67 publicações para os descritores nos bancos de dados já mencionados, desses, 53 apresentavam textos na integra, dos quais 16 foram publicados em português e 02 em espanhol, dos quais apenas 10 adequaram-se ao período de publicação e apresentaram títulos condizentes com os descritores, sendo assim incluídos na amostra.

Os 10 artigos incluídos serão descritos a seguir no Quadro 1:

Quadro 1: Caracterização da amostra do estudo. Juazeiro do Norte, CE.

\begin{tabular}{|c|c|c|c|}
\hline ACHADO & AUTOR/ANO & OBJETIVO & AMOSTRA \\
\hline 01 & $\begin{array}{l}\text { MANGANELLI } \\
\text { et al., } 2019\end{array}$ & $\begin{array}{l}\text { Caracterizar a população estudada e } \\
\text { descrever as intervenções dos } \\
\text { enfermeiros para a prevenção de } \\
\text { lesão por pressão em uma Unidade } \\
\text { de Terapia Intensiva Adulto. }\end{array}$ & $\begin{array}{l}\text { Pesquisa transversal descritiva, com } 13 \\
\text { enfermeiros de uma Unidade de Terapia } \\
\text { Intensiva, entre agosto e setembro de } 2017 . \\
\text { Foram utilizados questionário e estatística } \\
\text { descritiva. }\end{array}$ \\
\hline 02 & $\begin{array}{l}\text { CAUDURO et al., } \\
2018 .\end{array}$ & $\begin{array}{l}\text { Conhecer a atuação dos enfermeiros } \\
\text { no cuidado aos pacientes com lesões } \\
\text { de pele. }\end{array}$ & $\begin{array}{l}\text { Entrevistas com roteiro semiestruturado com } \\
21 \text { enfermeiros, analisadas pela Técnica de } \\
\text { Análise de Conteúdo na modalidade Análise } \\
\text { Temática. }\end{array}$ \\
\hline 03 & $\begin{array}{l}\text { BUSANELLO et } \\
\text { al., } 2015 \text {. }\end{array}$ & $\begin{array}{l}\text { Identificar os cuidados de } \\
\text { enfermagem para a prevenção das } \\
\text { lesões cutaneomucosas em pacientes } \\
\text { adultos hospitalizados e possíveis } \\
\text { dificuldades encontradas pelos } \\
\text { profissionais para promover estas } \\
\text { ações. }\end{array}$ & $\begin{array}{l}\text { Entrevista de } 118 \text { profissionais de enfermagem } \\
\text { atuantes nas unidades de internação clínica e } \\
\text { cirúrgica, unidade de terapia intensiva e pronto } \\
\text { socorro, de um hospital do Rio Grande do Sul. }\end{array}$ \\
\hline 04 & LIMA et al., 2017. & $\begin{array}{l}\text { Avaliar, com base no protocolo do } \\
\text { Ministério da Saúde, como a equipe } \\
\text { de enfermagem da UTI de um } \\
\text { hospital de Sergipe aplica às } \\
\text { medidas de prevenção contra a } \\
\text { formação de LPP. }\end{array}$ & $\begin{array}{l}\text { A população do estudo foi constituída por } 89 \\
\text { profissionais, os quais foram abordados e } \\
\text { convidados a participarem do estudo, porém } \\
\text { alguns recusaram o convite, constituindo } \\
\text { assim, uma amostra de } 70 \text { participantes. }\end{array}$ \\
\hline 05 & $\begin{array}{l}\text { SOUSA et al., } \\
2019 \text {. }\end{array}$ & $\begin{array}{l}\text { Identificar o conhecimento dos } \\
\text { enfermeiros assistenciais quanto à } \\
\text { prevenção e aos cuidados com lesões } \\
\text { por pressão (LPP) em unidades de } \\
\text { clínica médica e cirúrgica de um } \\
\text { hospital universitário de Brasília. }\end{array}$ & $\begin{array}{l}\text { Os dados foram coletados por meio da } \\
\text { aplicação de um instrumento com informações } \\
\text { acerca da formação e conhecimento do } \\
\text { enfermeiro acerca da LPP. A amostra foi de } 38 \\
\text { enfermeiros. }\end{array}$ \\
\hline 06 & $\begin{array}{l}\text { GALVÃO et al., } \\
2017\end{array}$ & $\begin{array}{l}\text { Descrever e analisar os } \\
\text { conhecimentos da equipe de } \\
\text { enfermagem acerca da classificação, } \\
\text { avaliação e medidas de prevenção de } \\
\text { úlceras por pressão (UP), em } \\
\text { pacientes internados na UTI de um } \\
\text { Hospital Universitário da cidade de } \\
\text { Manaus. }\end{array}$ & $\begin{array}{l}\text { Os dados foram coletados por meio de um } \\
\text { instrumento validado. Amostra de } 40 \\
\text { funcionários, sendo } 14 \text { enfermeiros e } 26 \\
\text { técnicos/auxiliares de enfermagem. }\end{array}$ \\
\hline 07 & $\begin{array}{l}\text { CARDOSO et al., } \\
2019 .\end{array}$ & $\begin{array}{llll}\text { Avaliar o } & \text { conhecimento } & \text { dos } \\
\text { enfermeiros } & \text { sobre Lesão } & \text { por } \\
\text { Pressão. } & & & \end{array}$ & $\begin{array}{l}\text { Realizado com } 26 \text { Enfermeiros que compõem } \\
\text { o quadro funcional de todos os setores de um } \\
\text { Hospital Privado em Teresina-PI. }\end{array}$ \\
\hline
\end{tabular}




\begin{tabular}{|c|c|c|c|}
\hline 08 & $\begin{array}{l}\text { CORREIA et al., } \\
2019 .\end{array}$ & $\begin{array}{l}\text { Verificar a prática referente à } \\
\text { avaliação da pele e do risco de } \\
\text { desenvolvimento de lesão por } \\
\text { pressão (LP) nos pacientes. }\end{array}$ & $\begin{array}{l}\text { Estudo descritivo exploratório com abordagem } \\
\text { quantitativa, realizado nas Clínicas: Médica, } \\
\text { Cirúrgica e UTI de Hospital de Ensino em João } \\
\text { Pessoa/PB. Realizado com a equipe de } \\
\text { enfermagem por meio de um questionário } \\
\text { estruturado. }\end{array}$ \\
\hline 09 & $\begin{array}{l}\text { MENDONÇA et } \\
\text { al., } 2018 .\end{array}$ & $\begin{array}{l}\text { Descrever as ações de enfermagem } \\
\text { prescritas por enfermeiros para a } \\
\text { prevenção de lesões por pressão e } \\
\text { sua ocorrência em centros de terapia } \\
\text { intensiva. }\end{array}$ & $\begin{array}{l}\text { Estudo transversal, descritivo e analítico, de } \\
\text { abordagem quantitativa conduzido em duas } \\
\text { instituições hospitalares de ensino de Campo } \\
\text { Grande, Brasil. A coleta ocorreu entre março e } \\
\text { junho de } 2016 \text { com } 104 \text { participantes. }\end{array}$ \\
\hline 10 & $\begin{array}{l}\text { SOARES et al., } \\
2018 .\end{array}$ & $\begin{array}{l}\text { Apresentar a aplicabilidade da } \\
\text { Escala de Braden na percepção dos } \\
\text { enfermeiros da atenção primária, e } \\
\text { identificar as medidas de prevenção, } \\
\text { e promoção da saúde de modo evitar } \\
\text { o desenvolvimento da lesão por } \\
\text { pressão. }\end{array}$ & $\begin{array}{l}\text { Pesquisa convergente assistencial, realizada } \\
\text { num distrito sanitário do Sul do Brasil, com } 20 \\
\text { enfermeiros. A coleta de dados ocorreu em } \\
\text { julho de } 2014 \text {. }\end{array}$ \\
\hline
\end{tabular}

Fonte: Elaboração própria dos autores.

A partir desses artigos utilizados, pode se notar a importância do conhecimento e da assistência do profissional de enfermagem frente ao paciente portador de LPP. Alguns principais achados são descritos a seguir:

Quadro 2. Principais achados dos estudos incluídos na amostra.

\begin{tabular}{|c|c|}
\hline $\mathrm{N}^{\circ}$ DO ACHADO & CONSIDERAÇÕES FINAIS \\
\hline 01 & $\begin{array}{l}\text { Os cuidados apontados com maior frequência compreenderam a avaliação da atividade-mobilidade dos } \\
\text { pacientes e o exame físico em sua admissão. Como cuidados preventivos, os enfermeiros prescreveram } \\
\text { a manutenção do paciente com pele hidratada, alternância de decúbito, higiene corporal e a utilização } \\
\text { de colchão piramidal. }\end{array}$ \\
\hline 02 & $\begin{array}{l}\text { Considerou-se o aprimoramento do enfermeiro, pela educação permanente, pelo trabalho em equipe e } \\
\text { pelo auxílio da Comissão de Prevenção e Tratamento de Feridas, uma estratégia para o cuidado da pele, } \\
\text { analisando as condutas dos enfermeiros e direcionando a prática profissional para o cuidado coletivo. }\end{array}$ \\
\hline 03 & $\begin{array}{l}\text { Os cuidados realizados para a prevenção de lesões cutaneomucosas em sua maioria estão de acordo } \\
\text { com o que a literatura preconiza. Contudo, o significado de segurança do paciente está restrito aos } \\
\text { cuidados com a prevenção de quedas e a prevenção das úlceras por pressão. }\end{array}$ \\
\hline 04 & $\begin{array}{l}\text { Os entrevistados que demonstraram conhecer algumas medidas, e informaram utilizá-las no } \\
\text { desenvolvimento de suas atividades. Revelou-se ainda que parte da equipe possuísse conhecimento de } \\
\text { algumas medidas para evitar o surgimento e agravamento de LPP. }\end{array}$ \\
\hline 05 & $\begin{array}{l}78.9 \% \text { acertaram entre } 70 \text { a } 89 \% \text { do instrumento, e somente dois enfermeiros }(5,2 \%) \text { obtiveram nota } \\
\text { igual ou maior a } 90 \% \text { de acerto. Os itens de menor acerto estão relacionados ao uso de dispositivos, } \\
\text { como luva d'agua }(23,6 \%) \text {, almofadas }(23,6 \%) \text {, e em relação a posicionamento e reposicionamento, } \\
\text { além da massagem em proeminências ósseas. }\end{array}$ \\
\hline 06 & $\begin{array}{l}\text { As médias globais de acertos foram } 63,4 \% \text { para os técnicos/auxiliares e } 51,4 \% \text { para os enfermeiros, } \\
\text { com diferença estatisticamente significativa entre os grupos somente para a categoria de prevenção de }\end{array}$ \\
\hline
\end{tabular}




\begin{tabular}{|c|l|}
\hline $\mathbf{0 7}$ & $\begin{array}{l}\text { UP. Déficit de conhecimentos sobre a prevenção de UP entre enfermeiros e técnicos/auxiliares de } \\
\text { enfermagem, tornando mandatória a capacitação desses profissionais. }\end{array}$ \\
\hline $\mathbf{0 8}$ & $\begin{array}{l}\text { Quanto aos acertos, } 74 \% \text { dos enfermeiros acertaram entre } 80 \text { a } 90 \% \text { do teste, } 9 \% \text { dos profissionais } \\
\text { acertaram entre } 60 \text { e } 69 \% \text {, sendo que } 9 \% \text { dos profissionais acertaram entre } 70 \text { e } 79 \% \text { do teste, } \\
\text { demonstrando falhas de conhecimento nos itens abordados, } 9 \% \text { acertaram acima de } 90 \% \text { onde estes } \\
\text { demonstraram conhecimento desejado em relação aos assuntos do teste. }\end{array}$ \\
\hline $\mathbf{0 9}$ & $\begin{array}{l}\text { Os principais resultados apontam para uma conformidade entre as ações e as recomendações da } \\
\text { literatura, no entanto acredita-se que é necessário investir em educação permanente sobre atualidades } \\
\text { em coberturas/curativos, para que os profissionais de enfermagem tenham um embasamento científico } \\
\text { mais significativo que permita atuar com segurança. }\end{array}$ \\
\hline $\mathbf{1 0}$ & $\begin{array}{l}\text { As ações de enfermagem apresentaram associações estatisticamente significativas quanto à ausência } \\
\text { de LP, o que evidencia a sua importância nos cuidados preventivos dessas lesões. No entanto, as ações } \\
\text { de enfermagem prescritas foram aleatórias e não atenderam às necessidades individuais do cliente ou } \\
\text { às baseadas na avaliação de risco. }\end{array}$ \\
\hline $\begin{array}{l}\text { Conclui-se que uma avaliação adequada, um plano de cuidados que possa prevenir a lesão por pressão, } \\
\text { assim como práticas que promovam saúde, configuram-se como possibilidades criativas versus } \\
\text { desafios, na inclusão de um novo paradigma na atenção primária. }\end{array}$ \\
\hline
\end{tabular}

Fonte: Elaboração própria dos autores.

\section{Discussão}

Considerando a Norma Técnica $\mathrm{n}^{\circ} 03$ do Ministério da Saúde (2017), destaca-se o uso de escalas para avaliar o risco de lesão por pressão para posteriormente realizar as práticas preventivas. Entre as escalas preditivas, é citada a escala de Braden, a qual envolve fatores intrínsecos e extrínsecos, como fricção e cisalhamento, umidade, mobilidade, nutrição, atividade e percepção sensorial.

Através dos achados de Lima et al (2017) foi possível evidenciar que os profissionais do estudo utilizam a escala de Braden ou Procedimentos Operacionais Padrões do próprio setor como protocolo para a prevenção de lesão por pressão nos pacientes internalizados.

Soares \& Heidemann (2018) também discorrem sobre a utilização da escala de Braden, considerando-a um aliado para a enfermagem, de forma com que proporciona um cuidado sistematizado e garanta um cuidado adequado. Os profissionais apresentaram aceitabilidade da utilização da mesma e expressaram maior segurança ao possuírem uma fonte segura e de fácil uso, possibilitando e facilitando um norteamento para cada caso.

Todavia, Lima et al (2017) citam ainda o conhecimento apresentado pelos enfermeiros, relatando ineficiência por mais da metade dos profissionais de enfermagem e demonstrando falta de conhecimento e preparo.

Confirmando isso, Sousa \& Faustino (2019) reafirmam um conhecimento insuficiente por parte dos enfermeiros e apontam a necessidade de capacitações, atualizações constantes e do interesse na busca por informações sobre o assunto.

Seguindo a mesma linha de pensamento, Cardoso et al. (2019) mencionam novamente resultados insatisfatórios em relação ao conhecimento do profissional enfermeiro sobre a prevenção dessas lesões, apresentando deficiência na base teórica e prática, os quais não souberam citar as escalas utilizadas, práticas preventivas ou tempos de mudanças de posicionamento e reposicionamento.

Galvão et al (2017) apresentam resultados fragmentados dos profissionais da enfermagem, entre enfermeiros e técnicos de enfermagem. Onde, se repete achados de conhecimento insuficiente e resultados baixos. Na segmentação entre as duas categorias, os técnicos de enfermagem apresentaram resultados superiores em todos os quesitos e questões, evidenciando diferenças significativas. 
Contradizendo o que foi apresentado, Correia \& Santos (2019) relatam maior feito pelo profissional enfermeiro, sendo superior no quesito de conhecimento anterior da escala de Braden, técnicas para prevenção, coberturas tópicas de caráter preventivo e dos cuidados utilizados para a limpeza diária da ferida.

É evidenciada uma necessidade de reconhecimento multidisciplinar, incluindo a equipe de enfermagem, o médico, o nutricionista, o fisioterapeuta, o psicólogo, variando e levando em consideração a necessidade do paciente específico, de forma com que, a equipe trabalhe em coletivo, com boa comunicação.

Diante o exposto, Cauduro et al (2018) apresentam achados sobre o trabalho em equipe e como este pode ser utilizado estrategicamente para o cuidado, se bem usufruído, para um cuidado contínuo que abranja todas as áreas. Entretanto, apresenta também os problemas que acompanham o trabalho em grupo, como divergências entre as condutas sem comunicação.

Observa-se que a prevenção de lesão por pressão carece de estrutura física adequada, equipe multidisciplinar qualificada e capacitada e dos recursos necessários, de forma com que, as medidas sejam adequadas para todas as necessidades apresentadas por cada paciente, de forma com que os cuidados gerais sejam realizados, mas que também sejam consideradas ações individualizadas.

Como exemplo de situações preventivas de lesões por pressão, Soares \& Heidemann (2018) tangem áreas de limpeza, higiene, hidratação, nutrição, inspeção e proteção. Citaram então, a redistribuição da pressão do corpo, lençóis limpos, alimentação balanceada, hidratação eficaz, o apoio familiar e emocional, educação em saúde e orientações para os pacientes, familiares e cuidadores sobre a temática.

Os achados de Mendonça et al (2018) aludem as ações frequentemente escolhidas por enfermeiros para prevenção de LPP, apresentando-se em maior prevalência a mudança de decúbito a cada 2 horas, seguido por outras prescrições como o uso de coberturas (hidrocoloide, película transparente), emolientes com ácidos graxos essenciais (AGE) para hidratação, coxins para reduzir a pressão nas proeminências ósseas, inspeção da pele, manutenção da higiene e manutenção da cabeceira a $30^{\circ}$, além da escala de Braden.

Reiterando tais práticas, Farias et al (2019) discorrem sobre o relato dos profissionais sobre as medidas utilizadas pelos mesmo, afirmando a mudança de decúbito, uso de óleos e hidratantes corporais, higiene e a proteção das áreas proeminentes do corpo que recebem pressão constantemente, que se encontram prejudicadas pela vascularização diminuída e a oclusão de vasos, dificultando a manutenção de uma boa circulação sanguínea.

Além das intervenções de enfermagem já relatadas anteriormente, Manganelli et al (2019) dispõem de outras ideias a serem consideradas, como a avaliação completa e contínua do paciente, a existência de fatores de risco ou predisposições e a criação e implementação de um plano de cuidados próprio de cada paciente, considerando seus problemas de saúde, doenças e os fatores de risco que o mesmo apresenta, baseando sempre o cuidado em conhecimento científico, conhecimento teórico e prático e protocolos da unidade.

Através dos achados discutidos, fica esclarecido que as práticas da assistência de enfermagem reduzem os números das lesões por pressão, sendo tratadas principalmente através da prevenção de riscos e do tratamento curativo do paciente. Porém, em contraste a isso o alto número de prevalências de casos ainda encontrados, comprova a necessidade de novas pesquisas acerca da prevenção (Soares, 2018).

\section{Conclusão}

Posto isso, nota-se a importância da assistência qualificada do enfermeiro baseada não somente no conhecimento empírico, mas principalmente no contexto científico, mantendo-se atualizado para um melhor entendimento sobre a forma de prevenir e tratamento de uma LPP, de maneira com que não ocorra percas acima do normal para a família e para o paciente que necessita desses cuidados. 
Por fim, enfatiza-se a importância de uma assistência qualificada e padronizada de forma que vá oferecer a melhor assistência possível e se atentando à procura do conhecimento e as mudanças que se fazem necessárias no atendimento para o paciente portador dessa patologia.

\section{Referências}

Adriani, P. A., Paggiaro, A. O., Ferreira, M. C., \& Carvalho, V. F. de. (2019). Aplicação do pressure ulcer knowledge test em enfermeiros de um hospital de atenção secundária - estudo transversal. Revista Enfermagem Atual In Derme, 87(25).

Almeida, F., Costa, M. M. S., Ribeiro, E. E. S. et al. (2019). Assistência de enfermagem na prevenção da lesão por pressão: uma revisão integrativa. Revista Eletrônica Acervo Saúde, n. 30, p. e1440, 14 ago.

Brandão, M. G. S. A., Brito, O. D. \& Barros, L. M. (2018). Gestão de riscos e segurança do paciente: mapeamento dos riscos de eventos adversos na emergência de um hospital de ensino. Revista de Administração em Saúde, São Paulo, v.18, n.70.

Busanello, J., Pinto, D., Schons, E., Baumgart, D., \& Poll, M. (2015). Cuidados de enfermagem ao paciente adulto: prevenção de lesões cutaneomucosas e segurança do paciente. Revista de Enfermagem da UFSM, 5(4), 597 - 606.

Cauduro F. P., Scheneider S. M. B., Menegon D. B. et al. (2018). Atuação dos Enfermeiros no Cuidado das Lesões de Pele. Rev. Enferm. UFPE on line., Recife, 12(10):2628-34, out.

Cardoso D. S., Carvalho F. M. O., Rocha G. B. et al. (2019). Conhecimento dos Enfermeiros sobre Classificação e Prevenção de Lesão por Pressão. Rev. Fund. Care. Online. 2019. abr./jun.; 11(3):560-566.

Correia A.S.B. \& Santos I.B.C. (2019). Lesão por Pressão: Medidas Terapêuticas Utilizadas por Profissionais de Enfermagem. Revista Brasileira de Ciências da Saúde. Volume 23 Número 1 Páginas 33-42.

Farias, A. D. A., Leal, N. T. B., Travassos, N. P. R. et al. (2019). Ocorrência de lesões por pressão em unidade de terapia intensiva de um hospital universitário. Revista Nursing, 2019; 22 (253): 2927-2931.

Galvão N. S., Serique M. A. B., Santos V. L. C. G. \& Nogueira P. C. (2017). Knowledge of the nursing team on pressure ulcer prevention. Rev. Bras. Enferm. [Internet]. 2017; 70(2): 294-300.

Lima, N. C. S., Oliveira, S. J., Nunes M. M. et al. (2017). Avaliação da assistência de enfermagem na prevenção de lesão por pressão em unidade de terapia intensiva. International nursing congress. Sergipe.

Manganelli R. R., Kirchhof R. S., Pieszak G. M. et al. (2019). Nurses' interventions in preventing pressure injury in an intensive therapy unit. Journal of Nursing UFSM. 2019. vol. e41: pi-p21.

Mendonça P. K., Loureiro M. D. R., Frota O. P. et al. (2018). Prevenção de lesão por pressão: ações prescritas por enfermeiros de centros de terapia intensiva. Texto contexto - enferm. Florianópolis, v.27, n.4.

Ministério da saúde (BR), Agência nacional de vigilância sanitária. nota técnica gvims/ggtes Nº3/2017: Práticas seguras para prevenção de lesão por pressão em serviços de saúde. Brasília; 2017.

Nunes R. O., Moreira K. C. C. \& Simon K. C. (2019). O enfermeiro e a sistematização da assistência ao paciente com Lesão Por Pressão. REVISTA UNINGÁ, [S.1.], v. 56, n. S6, p. 68-75, set. 2019.

Oliveira V. C. \& Constante S. A. R. (2018). Lesão Por Pressão: uma revisão de literatura. Psicologia e Saúde em debate, v. 4 , n. 2 , p. $95-114,18$ jul. 2018.

Santos A. L. F., Oliveira B. V. B., Palmeira N. M. S. et al. (2018). A Escala de Braden como Protocolo de Prevenção de Lesões Por Pressão: Uma Revisão Integrativa. Ciências Biológicas e de Saúde Unit, Alagoas v. 5, n. 1, p. 193-204, novembro, 2018.

Silva C. R. L., Araújo C. S. B., Alves S. M. et al. (2019). A enfermagem na atenção domiciliar e os desafios do cuidado ao portador de lesão por pressão. Enfermagem Moderna: Bases de Rigor Técnico e Científico 3, 2019. Cap. 1, pag. 1-12.

Soares C. F. \& Heidemann, I. T. S. B. (2018). Promoção da saúde e prevenção da lesão por pressão: expectativas do enfermeiro da atenção primária. Texto contexto-enferm. Florianópolis, v.27, n.2, e1630016, 2018.

Sousa R. C. \& Faustino A. M. (2019). Conhecimento de enfermeiros sobre prevenção e cuidados de lesão por pressão. J. Res. Fundam. Care. Online 2019 jul/set 11(4): 992-997.

Souza, M. T. de., Silva, M. D. da. \& Carvalho, R. (2010). Revisão integrativa: o que é e como fazer. Einstein (São Paulo), 8(1), $102-106$. 